\title{
Geistliche Umdichtung weltlicher Lieder.
}

Die Contrafactur weltlicher Lieder, wie man es im sechzehnten Jahrhundert nannte, ist eine durch die romanische und deutsche Lyrik des Mittelalters sich hindurchziehende litterarische Erscheinung. Viele dieser Lieder können wir als Parodien bezeichnen, die ihr Gegenstück in den zum Teil recht frechen Umdichtungen geistlicher Lieder haben, aber in den meisten Fällen bediente man sich der Anknüpfung an das weltliche Lied nur, um durch dessen populäre und vielgesungene Melodie das geistliche Lied zu verbreiten.

Einen nicht unwichtigen Beitrag zur Geschichte der Umdichtungen liefern die geistlichen Lieder einer Pariser Handschrift, auf welche schon Jubinal, Nouveau Recueil II 4I3-423 hingewiesen hat, die ihrem lyrischen Inhalte nach jedoch erst durch G. Raynaud in seiner eben erschienenen 'Bibliographie des Chansonniers français des $\mathrm{XIII}^{e}$ et XIV ${ }^{e}$ siècles' (Paris 1 884) erschlossen worden ist. Als ich in diesem Frühjahr zur Vervollständigung meiner seit Jahren vorbereiteten Sammlung altfranzösischer volksmälsiger Refräns die Liederhandschriften der Pariser Bibliotheken durchforschte, machte mich G. Raynaud auf die Handschrift fr. 12483 aufmerksam, die in dem grölsten Teil der in ihr enthaltenen geistlichen Lieder den Refrän verwendet. Das Verzeichnis derselben findet man Bibliogr. I $149 \mathrm{f}$. zuteilen. ${ }^{1}$

Es möge gestattet sein die bemerkenswertesten davon hier mit-

I.

A la virge qui digne est de s'amour, envoia diex son angre Gabriel.

'di li', fait il, 'un salu de doucour

et qu'envoier li vuel un don nouvel,

5 moy meismes: je n'ay plus bel jouel.

or soit joians, si lait toute tristour,

car de lui chant une chancon sans plour

que je ne chant de nule autre pucele:

Se j'ai ame, j'ai choisi

10 du mont la plus bele.

I. Bl. $3^{\mathrm{d}} \cdot 6$ ioian.

1 Die durch Beschneiden und Verstümmelung der Blätter weggefallenen und von mir ergänzten Buchstaben sind kursiv gedruckt. 
Cele doit bien de cuer joie mener qui puet dire "j'ai eslit le melliour et le plus bel c'om puist jamez trouver, cui $j$ 'ai donne et mon cuer et m'amour;

15 et se je l'aim, il m'a fet tel honnour qu'il me daigne par amour saluer et dit qu'il veut en mon cors reposer:

si sui joians du salut qu'il me mande.

Puis que diex fait de mon cuer fin sa chambre, joie en ai grant, que reson le commande."

Je ai de mon ciel veu humilite et le douz cuer que a sans repentir et la vertu de sa virginite et le talent qu'el'a de moy servir,

25 si qu'ele est diex, que nul'autre aatir ne se pourroit a lui par dignite, ne que l'estoile se peut par biaute ne par clarte comparer a la lune.

Je n'i voy cui je doie amer fors une.'

30 'Hainc n'oi talent d'autre amour acointier ne volente de cestui deguerpir, hainz l'aim et serf tousjors de cuer entier, qu'a lui doit bien tous li mons obeir, et se je l'aim, je le doi bien servir,

35 qu'il m'a donne plus de mon desirrier. en lui doit l'en son amour emploier, car autre amour ne fet fors devoir.

Se de lui ne me vient joie, d'autrui ne la quier avoir.'

40 'Ma genz pour cui ai soufert grief tourment, painne de mort pour vostre mort tourner, se vous m'amez', fet il, 'ne tant ne quant, donc vous pri je de ma mere honourer, qu'ele vous puet si bien guerredonner

45 comme cele qui puet tout son talent faire de moi comme de son enfant. se vous voulez en moy merci trouver,

$\mathrm{Ne}$ vous repentez mie de ceste dame amer.'

32 touriors. 35 desirrer. 37 fehlt eine Silbe. 40 Sa.

Dem Liede gehen folgende Verse voraus:

pren moy, compaigne, par la main et chanton de la pucelete

- ceste petite chanconnete; 
also ein Lied, das zur Begleitung des Tanzes gesungen werden soll. Der Refrän der ersten Strophe ist ein wohl bekannter: er wird verwendet in einer anonymen Pastourelle (meine R. u. P. II I6, Io) und ebenso von Baudouin de Condé, Prison d'amour V. 2580 . Er wird dadurch als ein volksmärsiger erwiesen.

Auch der Refrän der dritten Strophe ist anderweitig zu belegen: in einem anonymen Salut d'amour bei Jubinal, Nouveau Recueil II 237.

Der Refrän der letzten Strophe ist die Umgestaltung cines weltlichen, der lautet

\section{$\mathrm{Ne}$ vos repentez mie}

de loiaument amer,

und den ich nicht weniger als fünf mal nachweisen kann: bei Baudes de la Kakerie (R. u. P. I 7I, 33); bei Pierre de Corbie (R. u. P. III 33, 66); in der Cour de paradis V. 270; im Guillaume de Dole (Jahrbuch II, I62), und bei Colart le Bouteillier in der sechsten Strophe des Liedes 'Je ne sai tant merci crier' (fr. I 26 I5, Bl. $24^{\mathrm{a}}$ ).

Ganz ersichtlich ist auch der Refrän der vierten Strophe ein dem Volksliede entlehnter, wenn er auch nicht anderweitig belegbar ist. In der zweiten Strophe hat der Refrän eine Umgestaltung in geistlichem Sinne erfahren: den Wortlaut des weltlichen sind wir nicht in der Lage herzustellen.

II.

Agniaus dous, agnias gentis, agniaus sans tache, agniaus qui pour nous geutes en la creche, agniaus . . . . . . . . . . . . .

agniaus, pour vous hoy tant duel c'onques je sache

5 ne n'oy tant.

Qui me rendroit mon aigniel et mon damache, a lui me rent.

Li lous prist pes a l'aigniel dous debonnaire:

ce fu Judas li trahitres deputaire,

Io qui aus juis le bailla pour tel affaire. honni soient tuit li lou de tel repaire outreement.

Qui me rendroit mon aigniel et mon damache, a lui me rent.

I5 Honni soient lou prive et lou sauvache! je ne vi onques bon leu en mon eage. lou sont cruel par nature et plain de rage: que que li autre ont fait, cil m'a damache fet trop grant.

20 Qui me rendroit mon aigniel et mon damache, a lui me rent.

II. Bl. $25^{\mathrm{c}}$ 3 eine Zeile ist weggeschnitten. Io au. 
Il est plus de lous u siecle que d'agniaus:

la char menjuent et boivent sor les piaus;

li riche les povres metent aus fuisiaus.

25 helas de ce qu'e a faire li miens diaus

le cuer me fent.

Qui me rendroit mon aigniel et mon damache, a lui me rent.

Agniaus dous, plus dous, tres dous, douce couree,

30 agniaus, comment remaing seule et esgaree!

agniaus, comment seray mes reconfortee?

mors, quar vien et si m'oci guele baee

et plus n'atent.

Qui me rendroit mon aigniel et mon damache,

35

a lui me rent.

224 au. 25 mien duel. 32 baes.

Dafs der Refrän auch dieses Liedes entlehnt ist, beweist sein Vorkopmmen in einem Salut d'amour, Jubinal II 24I. Hier wird er dunrch die Worte eingeleitet: com cele qui chanta cest motet en presennt, eine Bezeichnung, die sich vor Refräns nicht selten findet und ddie sich am natürlichsten daraus erklärt, dals in Motets die Refränns volksmärsiger Lieder ebenfalls häufige Verwendung fanden. Das Liied, zu welchem der Refrän gehört, schilderte die in der Pastouurelle mehrfach vorkommende Situation, dafs der Schäferin von ddem Wolfe ihr Lamm geraubt wird; sie verspricht dem, der es ihr - wiederbringt, ihre Liebe. Das Versmals unseres Liedes ist der boekannte elfsilbige Vers mit Cäsur nach der siebenten, weiblich naach der achten Silbe. Die kurze Zeile, die die fünfte Zeile der Sttrophe bildet, kann drei und vier Silben haben: letzteres nur, wenn : sie vokalisch anlautet und die vorausgehende vokalisch schliefst ( 1 2. 333), oder wenn die vorhergehende Zcilc stumpf reimt (25).

III.

L'autrier matin el moys de may

regis eterni munere

que par un matin me levay

mundum proponens fugere.

5 en un plesant pre m'en entray

psalmos intendens psallere:

la mere dieu ilec trouvay

jam lucis orto sidere.

Grant painne mist en lui fourmer

sol et nature studium:

IIII. Bl. $54^{\circ} . \quad 3$ von dem $\mathrm{m}$ von me ist nur ein Drittel vorhanden. 
de sa biaute vont raconter

l'en ne pourroit son per trouver

in numero mortalium:

15. Sa puissance vout esprouver

deus creator omnium.

Cler out le vis et le cors gent

divino moderamine

comme la rose entre la gent

20 in gemmis grato temigne.

plus que cristal sont blanc si dent, recte loquentur ordine.

n'a tant bel jusqu'en occident

a solis ortus cardine.

25 Par le pre vait soutivement celesti plena gracia.

vers lui me trais isnelement

ejus sequens vestigia,

et li dis 'bele, a vous me rent

30 quam amo super omnia, qu'en vous habite apertement

eterni celi gloria.'

Quant je la vi si seule aler et progressu deifico,

35 je regarday son dous vis cler vultu satis angelico:

lors vins a lui sans demourer cum affectu pacifico.

la commencay a saluer

40 ex more docti mistico.

'Douce dame, le haut seignour celum regens empireum soit o vous par sa grant doucour, flos et decus virgineum.

45 vostre homme soi sans nul retour:

hic super florem croceum

conseliez cest las pecheour:

ecce tempus ydoneum.

Secoures moy et conseiliez,

50 parens et felix femina,

11 de] das erhaltene scheint ein $\mathrm{n}$. 12 abgeschnitten, ein $\mathrm{s} 1$ sind erkennbar. 20 temigne? 22 rete. 23 bele iuqus en. 24 ortu". 25 voit. 31 hïte. 35 dous vis scheint durchstrichen. 45 soit. 
qui a la destre den seez

sanctorum super agmina.

dampnant immensa crimina,

55 se je ne sui par vous sauvez,

o gloriosa domina.'

El respont 'de tout mal garder

te velit dei filius

qui de moy pour homme sauver

60 carnem sumpsit propicius.

saches, pour toy amonnester,

ut sit tibi salubrius,

me fait hui si matin lever

ales diei nuncius.

65 Fai bien et si lesse pechie quod dilexisti primitus;

de folie retrai ton pie crebretque pro me genitus. se cilz le fait, cilz sera lie

70 qui te dictavit celitus;

en ton cuer sert par ta pitie

veni creator spiritus.'

Quant enseignie m'eut et apris predulcis vena venie,

75 maintenant a ses piez me mis

demissa solo facie.

de bon cuer li proie et dis

'mater misericordie, par vous metes en paradis,

80 summe deus clemencie.'

Apres s'asist dessus la flour

colore pinctum vario;

si me moustra sa grant valour

omni carentem vicio.

85 je fui sans faille celui jour

tanto repletus gaudio

c'onques nus ne me fist gregnour

Jhesu nostra redempcio.

Les flours flairent plus que piment

90 quos aura levis ventilat;

dejouste nous joieusement

mitis alauda jubilat,

51 sees. 53 abgeschnitten. 55 sauues. 57 Ele. 68 cribret te per me genitus? 71 serf? 81 fleur. 92 mittis. 
et le roussignol doucement

blando garritu sibilat.

95 donc dist ma dame simplement

'aurora lucis rutilat.'

Repairier vout tout maintenant

ad dulces choros superum;

lors me fist el front en riant

100

signum crucis splendiferum,

et dist a vois clere et plaisant

'fili, largitor munerum,

cest mon devot serf te commant,

conditor alme syderum.'

105 Apres icest mot.sans mentir ascendit ad celestia:

je vi encontre ele venir sanctorum decem milia.

je qui remains ploure et souspir

I IO cordis tactus angustia, diex! verrai je james venir beata nobis gaudia?

Mere de dieu, vrai salut port, fons pietatis maxime,

115 de celui m'envoies confort salutem prestans anime. garde moy de l'anemi fort qui me temptat sepissime; paradis m'otroit a la mort 120 rerum creator optime.

amen.

Die Mischung lateinischer Verse mit romanischen oderdeutschen ist ebenfalls ein bekannter Zug der mittelalterlichen besic. Wie hier werden Anfangsverse und sonstige Stellen von lateinichen Hymnen mit Versen in der Volkssprache gemischt in einemrecht anstölsigen Gedichte, das in verschiedenen Fassungen vorhnden ist (Germania I 7, 188 ff. Fichards frankfurt. Archiv 3, 26o ff. Hoffmann von Fallersleben, In dulci jubilo S. $90 \mathrm{ff}$. Anzeiger für ’unde der deutschen Vorzeit I880 Sp. I 73 f.). In dem französischo Gedichte hat, wie schon der Anfang zeigt, die Pastourelle dasgeistlich umgewandte Motiv und die ganze Einkleidung hergeeben. Ein Gedicht von Gavaron Gratelle (R. u. P. I 69) beginnt 'L'utrier le premier jor de mai' und stimmt in der Strophenform mi dem geistlichen Liede überein; gleichwohl ist es nicht als das witliche Original desselben anzusehen, denn dann müfsten wörtlicb Anklänge vorhanden sein. 


\section{IV.}

Diex, comment pourrai

savoir la volente

ma douce dame, a cui j'ai

tout mon cuer donne?

5 Pour s'amour ai en dolour lonc temps este qu'onques ne li osai dire mon pense.

or li pri qu'ele m'i tiegne a amiste:

mort m'aroit s'ele n'avoit de moy pite.

Diex, comment pourrai

10

savoir la volente

ma douce dame, a cui j'ai

tout mon cuer donne?

Dame Marie, a toy me sui donne:

si forment avez mon cuer emprisonne,

15 quar ne puis avoir secours, que bien le se, fors de vous en cui repose ma sante.

Diex, comment pourrai

sav'oir la volente

20

$$
\text { ma douce dame, a cui j'ai }
$$

tout mon cuer donne?

Puis que mon cuer est pour vous si atourne qu'a nule autre fors qu'a vous n'ai mon pense, dame, aiez de moi merci en charite:

ou se ce non, certain sui que je morre.

Diex, comment pourrai

savoir la volente

ma douce dame, a cui j'ai

tout mon cuer donne?

Dame, pour vo biau semblant qu'avez monstre

30 a moy qui vous aim de cuer sans faussete,

cuide de vous a tousjors estre prive;

mes enhay m'avez tout pour mauveste.

Diex, comment pourrai

savoir la volente

35

$$
\text { ma douce dame, a cui j'ai }
$$

tout mon cuer donne?

Au definer de mon chant vous priere

que pour pechie je ne soie refúse, quar de tres fin cuer vous aim et amere

40. ne ja tant comme jegrvis n'en faussere.

IV. BL. 107 a. 13 um eine Sibe zu kurz. Vielleicht umsustellen me sui a toy. 32 pour ma mauvestie. 37 pri ie. 38 que ie ne soie pour pechie refuse. 


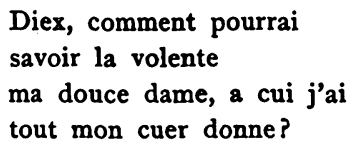

Der Refrän dieses Liedes kommt in einer anonymen Pastourelle der Handschrift $\mathrm{C}$ (R. u. P. II 42, 36) vor, mit geringer Abweichung: die Versabteilung ist anders als in meiner Ausgabe zu machen

$$
\begin{aligned}
& \text { Duez, coment porai } \\
& \text { savoir la volantei } \\
& \text { de vos, dame, a cui j'ai } \\
& \text { tot mon fin cuer donei, }
\end{aligned}
$$

und diese Lesart ist wohl die bessere. Die Versart der geistlichen Umdichtung ist wieder der elfsilbige Vers; schon danach ist $\mathrm{zu}$ vermuten, dafs ein volksmäfsiges weltliches Original zu Grunde liegt.

\section{V.}

Ave Maria, j'aim tant.

Pleust dieu, le filz Marie,

ne pucele ne beguine

5 qui n'amast dieu tendrement.

Ave Maria, j'aim tant.

La beguine s'est levee

de vesture bien paree:

au moustier s'en est alee,

10. Jhesu Crist va regretant.

Ave Maria, j'aim tant.

Quant ele vint a l'esglise,

jus a la terre s'est mise,

si vit dieu le filz Marie

i5 en crois pendant laidement.

Ave Maria, j'aim tant.

Apres ce s'est relevee

et l'ymage $a$ regardee

et les plaies ravisee:

20 $a$ poy le cuer ne li fent.

Ave Maria, j'aim tant.

Apres ce s'est escriee

'ha lasse maleuree

V. Bl.240a. 2 eine Zeile weggeschnitten. Vielleicht ne fust en toute sa vie. 7 zwischen beguine und sest ist ïbergeschrieben main. Ohne Zweifel hatte das weltliche Original s'est main levee, was aber den Vers hier zerstören würde. 8 von u in vesture ist noch eine Hälfte sichtbar. 
qui recevra tel colee

25 con le jour da jugement.

Ave Maria, j'aim tant.

Prestre clerc et chevalier, damoiseles, escuier, bourgois et gent de mexcier

30 i seront tuit en present.'

Ave Maria, j'aim tant.

$28 \mid \mathrm{le}^{8}$ escuier.

Deutlich ist hier noch in zahlreichen Wendungen und Ausdrücken das weltliche Original zu erkennen. Namentlich lälst sich vergleichen ein Fragment aus Guillaume de Dole (R. u. P. II I I 8)

Mauberjon s'est main levee,

dioree (?) buer i ving:

a la fontaine est alee

Vgl. 7. 9. Ein ähnlicher Refrän wie in unserm geistlichen Liede kommt in einer Pastourelle von Guillaume le Vinier (III 29) vor. Wenn man dort abteilt

dorenlot!

deus or haes, je l'aim tant,

so ist die zweite Zeile dem Refrän des geistlichen Liedes vollkommen gleich.

VI.

Du dous Jhesu souvent devons chanter et lire.

Chanter m'estuet, quar volente m'en prie,

du rossignol qui d'a... . . .

. . dous Jhesus

5 qui est monte lassus,

et nous sommes ca jus:

tous li cuers m'en souspire.

du dous Jhesu devons adez bien dire.

Du douz Jhesu souvent devons chanter et lire.

Io Rossignolet, bien faites vostre office:

les fins amans bien aprenez a vivre,

dites 'fuiez fuiez,

tout le monde laissiez,

ne vous $i$ apuiez,

15 quar trop i a de guile:

li dit Jhesu sont vrai com evangile.'

$\mathrm{Du}$ douz Jhesu souvent devons chanter et lire.

VI. $B l .243^{\text {d. }} 3$ rossignol $^{\text {let }}$ qui da $\mid z u$ ergänzen offenbar d'amer; vielleicht me mestrie. 4 ihesu. 8 ihu. 9 Der Refrän, der in dieser und den folgenden Strophen nur $\mathrm{Du}$ douz lautet, ist von jüngerer Hand beigefügt. 16 dist. 
Rossignolet, par vo grant cortoisie menez moy en o vous en la gaudie:

20 la serons en deduit et le jour et la nuit et si lorons celui qu'amours firent ocirre. folz est li cuets qui Jhesum ne desirre.

25 - Du douz Jhesu souvent devons chanter et lire.

De li loer ne se devroit nus taire: merveilles fist quant vint en no repaire. povres volt devenir et en la crois mourir

merveilles fu a faire.

diex du ciel vint pour nous d'enfer retraire.

Du douz Jhesu souvent devons chanter et lire.

Rossignolet, pour dieu, quar me conseille:

35 ou est li hons qui feist tel merveille, qui souffrist cruel mort

et si grant vilain tort pour rendre a nous confort qu'avons perdu la vie?

40 Jhesus est diex et filz dame Marie.

Du douz Jhesu souvent devons chanter et lire.

Rossignolet Jhesu de piteus estre, assie nous tous delez toy a ta destre, en ce biau paradis

45 qui est parez tous dis: la sont joie et delis. diex, tant $i$ fait bon estre. li douz Jhesus siet du pere a la destre.

Du douz Jhesu souvent devons chanter et lire.

50 La verront cil nostre dame Marie qui l'averont en lour vie servie de cuer et en purte:

la seront a seurte en pardurablete:

55 bele est sa compaignie. Jhesus nous doint que $j a$ n'i failons mie.

$\mathrm{Du}$ douz Jhesu souvent devons chanter et lire.

I9 en fehlt. 36 cruel fehlt. 37 vilain tort] vilēnie. 38 confort fehlt. 50 nostre dame fehlt. 51 en lour vie fehlt. $54{ }^{\text {en }}$ pardurable vie.

Der Refrän dieses Liedes lärst sich allerdings nicht als ein volkstümlicher nachweisen und ist jedenfalls durch den Umdichter 
stark verändert worden ${ }^{1}$; aber dafs auch hier der Umdichter sich an ein weltliches Original angeschlossen hat, ergiebt sich aus der Rolle, die der Nachtigall hier zugeteilt ist. Vgl. meine altfranzösischen Volkslieder S. xxxu f. Die Strophenform schliefst sich mit Ausnahme einer Zeile genau an das Lied von Gaces Brulez 'Quant bone dame et fine amors me prie' an.

\section{VII.}

Vous ne savez que me fist Jhesucrist li miens amis, qui Jacobine me fist par grant amours,

5 Li debonnaires. Il m'a si navre d'un dart, n'i $a$ la plaie qui pert: ja nul jour ne guarire se par li non.

Io $\mathrm{Li}$ debonnaires.

Diex! son dart qui m'a navre, comme il est dous et souefz! nuit et jour m'i fait penser com diex est douz,

\section{Li debonnaires.}

Quant regart par paradis donc $l i$ rois est mes amis, de lermes et de soupirs mes cuers font tous.

$20 \mathrm{Li}$ debonnaires.

Se je souvent plouroie et tres bien dieu amoie, il me donroit sa joie, autrement non.

$25 \quad L i$ debonnaires. Quant je pense a Marie, qui fu de nete vie, j'ai une jalousie que ne m'est bon.

$30 \mathrm{Li}$ debonnaires.

Prions donc la pucelle qui fu sainte et honneste qu'en paradis nous mete: c'est moult biau don.

$355 \quad \mathrm{Li}$ debonnaires.

VII. $B l .253^{\text {b. }} \quad 6$ nauree. $\quad 7$ m... la. 31 donc fehlt.

1 Vielleicht sogar um zwei Silben zu lang: ich denke, souvent wird zu streichen sein. 
Hier ist mir die Nachbildung eines weltlichen Originals zweifelhaft. Doch hat die Strophenform mit ihrem Refränreim als fünfte Zeile etwas volksmälsiges. Bemerkenswert ist der Wechsel des Reimgeschlechtes von der fünften Strophe an: die Silbenzahl bleibt unverändert. In der zweiten Strophe ist wohl nicht alles in Ordnung; Z. 8 wird wohl guarira zu schreiben sein, sc. la plaie.

\section{VIII.}

Diex, li dous dieus, au cuer ai amouretes, s'ameray.

He diex! pour quoy n'est bien amez

cilz qui souffri tant de doulours,

5 navres ou cuer et entamez,

batuz plaiez mors par amours?

- . . plain de doucour.

amez le, gentilz cuers, amez,

pensez a lui, dites tous jours:

Io amis, a vos maus penseray.

Diex, li dous dieus, au cuer ai amouretes, s'ameray.

VIII. Bl. $253^{\text {d. }} 4$ douleurs. 6 nach par ist eine Zeile weggeschnitten.

Der Refrän dieses Liedes, von welchem nur die erste Strophe erhalten ist, findet sich auch in einem Liede von Pierre de Borgne verwendet: ' $\mathrm{Li}$ rossignols que j'oi chanter' (Wackernagel, Altfranz. Lieder No. 30, anonym; Dinaux, Trouvères de la Flandre p. 348) in der Form

Deus, li dous deus, j'ai au cuer amoretes, s'amerai.

Etwas abweichend Hist. litt. 23, 689

He dex dex dex, j'ai au cuer amorete, s'amerai,

und in fr. 12615, Bl. $76^{b}$

E diex diex diex, j'ai au cuer amoretes, s'amerai.

Doch nicht blofs der Refrän stimmt, sondern die Strophenform ist identisch; so dafs in diesem Falle das bestimmte Original, das dem geistlichen Umdichter vorlag, nachgewiesen ist. Ob der Umdichter bei den folgenden Strophen auch den Refrän gewechselt hat, mufs unentschieden bleiben.

IX.

Amis, amis,

trop me laissiez en estrange pais.

L'ame qui quiert dieu de veraie entente souvent se plaint et forment se demente,

IX. Bl. 264 b. 3 Raynaud ergänzt de toute s'entente. 
5 et son ami cui venue est trop lente va regretant que ne li atalente.

Amis, amis, trop me laissiez en estrange pais.

Trop me laissiez $c i$ vous longuement querre

Io en cel regne et en mer et en terre. enclose sui en cest cors qui me serre de ceste char qui souvent me fait guerre.

Amis, amis, trop me laissiez en estrange pais.

15 Diex! donnez moy ce que mes cuers desirre, pour cui languis, pour cui sui a martire. Thesucrist est mes amis et mon sire, $l i$ biaus, li bons plus que nul ne scet dire.

Amis, amis,

20 trop me laissiez en estrange pais.

Mon createur, quar je sui sa faiture, qui me nourrit et de tout me procure, mes amis est, quar en moy mist tel cure et par amour se joint a ma nature.

25 Amis, amis, trop me laissiez en estrange pais.

Il m'apela ains que je l'apelasse, i1 me requist ainz qu'aprez lui alasse. or est bien droit qu'en lui querre me lasse,

30 qui que cest mont pour lui trouver trespasse. Amis, amis, trop me laissiez en estrange pais.

Et quant j'auray passe ceste bruine, ou li jour faut et le vespre decline,

35 cilz qui les cuers alume et enlumine me moustrera: lors auray joie fine.

Amis, amis, trop me laissiez en estrange pais.

6 ne zwischengeschrieben. 7 vom Refrän nur die erste Zeile, ebenso in den übrigen Strophen. 10 regnes. von $\mathrm{m}$ ein Drittel erhalten.

Ein besonders anziehendes Beispiel von Umdichtung. Denn offenbar war das weltliche Original eine jener volksmärsigen Romanzen, deren leider nur eine so kleine Zahl sich erhalten hat. Unter den erhaltenen sind zwei, mit denen dies geistliche Lied sich berührt. Im Refrän mit I 4

e amis!

por medissans seus fors de mon pais, 
wo die Strophenform aus drei Zehnsilblern mit männlichem Reime besteht. Noch gröfser ist die Übereinstimmung mit I 9, wo die Strophe aus vier Zehnsilblern mit weiblichem Reim besteht und der Refrän lautet

\section{ae cuens Guis amis!}

la vostre amors me tout solaz et ris.

Abgesehen also von der ersten Refränzeile, die in I 9 zwei Silben mehr hat, stimmt die Strophenform ganz genau. Auch im Ausdruck stimmt die Zeile I I mit 9, 9 qui en cest mes m'a mise et enserree. Gleichwohl ist I 9 nicht das Original; denn offenbar ist der Refrän unverändert aus demselben herübergenommen. Die im fremden Lande verlassene Geliebte, die ihren Geliebten herbeisehnt, ist ein echtes Romanzenmotiv. Der Refrän konnte um so eher beibehalten werden, als das estrange pais aufgefalst wird als die Erde, gegenüber dem Himmel, der ersehnten Heimat, wie in der altd. geistlichen Dichtung ellende (= estrange pais) die ganz gleiche Bedeutung hat. Mehreres von dem Wortlaut des Originals ist in den ersten Strophen ersichtlich und mit geringer Änderung läfst der Anfang sich herstellen. In der ersten Zeile war wahrscheinlich, wie gewöhnlich, der Name der Heldin genannt

$$
\begin{aligned}
& \text { Bele .... aime de voire entente, } \\
& \text { souvent se plaint et forment se demente } \\
& \text { et son ami cui venue est trop lente } \\
& \text { va regretant que ne li atalente. }
\end{aligned}
$$

Auch in der zweiten ist der Anschlufs an das Original noch ersichtlich: sie ist eingeschlossen, wie Belle Euriaus (I 16 seule est enclose), vielleicht Z. 12 de mes parens qui souvent me font guerre. Auch Z. 15. 17. 18 verraten noch die Anlehnung. Erst von der vierten Strophe an bewegt sich die Umdichtung freier. Wir haben hier also deutlich erkennbare Trümmer einer untergegangenen Volksromanze vor uns.

\section{$\mathrm{X}$.}

Li solaus qui en moy luist est mes deduis et diex est mes conduis.

Et que me demandez vous, amis mignos? quar $a$ vous ai tout donne et cuer et cors.

5 et que voulez vous de moy? voulez ma mort, savoreus Jhesucrist?

Li solaus qui en moy luist est mes deduis et diex est mes conduis.

X. Bl.266c. 3 Raynaud giebt als Anfangszeile an Et que me demauconduis. Allein das neben deman stehende conduis bildet den Schluss des als Thema vorangestellten Refräns. 5 voulez vous ma mort. 7.8 nur $\mathrm{Li}$ soulaus, von jüngerer Hand beigefügt, ebenso bei Strophe 3, aber bei 4.5 von der ersten Hand $\mathrm{Li}$ solaus, und bei $6 \mathrm{Li}$ solauz etc. 
Je li feray une tour a mon cuercon:

10 ce sera ou plus biau lieu de ma maison;

il n'en istra ja nul jour, mon ami douz, ains sera en deduit.

Li solaus qui en moy luist est mes deduis et diex est mes conduis.

15 Cil doit bien estre esbaudis qui sert tonsdis en fais et en dis le roy de paradis

Li solaus qui en moy luist est mes deduis et diex est mes conduis.

Diex! or ardent cil bisson par paradis: amors les font jubiler et tressaillir. fins amans ont tout le temps en Jhesu Crist, quar c'est tout leur desir.

25 Li solaus qui en moy luist est mes deduis et diex est mes conduis.

Je mi lasse, que feray? n'i puis aler. esperance et fine amour, quar m'i portez, qu'aprez ceste mortel vie $i$ puisse aler:

30 ce sont tous mes deduis.

$\mathrm{Li}$ solaus qui en moy luist est mes deduis et diex est mes conduis.

Dame Marie, priez a vostre fil que tant com nouz vivons en mortel essil,

35 sa grace nouz doint, par quoy soion si fil et en son livre escrit.

$\mathrm{Li}$ solacz qui en moy luist est mes deduis et diex est mes conduis.

9 nach tour ein Reimpunkt, ebenso II nach iour, doch sind die Cäsurreime wohl nicht beabsichtigt. 13. 14 der Refrïn fahlt hier: die beiden folgenden Zeilen sind an die sweite Strophe angeschlossen, nach 16 keine Lücke bezeichnet. 34 en ce mortel. 35 que sa grace.

Einen ähnlichen Refrän vermag ich nicht nachzuweisen: allein dafs auch hier ein weltliches Original zu Grunde liegt, wird einmal durch die Versart, den elfsilbigen volksmälsigen Vers, und dann durch den Inhalt der ersten Strophe wahrscheinlich gemacht; auch die zweite Strophe schliefst sich ersichtlich noch ihrem Vorbilde im Wortlaute an.

\section{K. BARTSCH.}

